\title{
Estimulando o pensamento computacional em alunos do ensino médio com o uso do Scratch for Arduino
}

\section{Alan Gomes Silva ${ }^{1}$, Ramásio Ferreira Melo ${ }^{2}$, Rogério Pereira de Sousa ${ }^{3}$, Karoline Araújo Nascimento ${ }^{1}$}

${ }^{1}$ Graduados em Licenciatura em Computação - Instituto Federal de Educação, Ciência e Tecnologia do Tocantins (IFTO), Campus Araguatins
${ }^{2}$ Mestre em Educação - UFSM. Professor EBTT de Informática - IFTO, Campus Araguatins
${ }^{3}$ Mestre em Engenharia de Produção e Sistema - UNISINOS. Professor EBTT de Informática - IFTO, Campus Araguatins

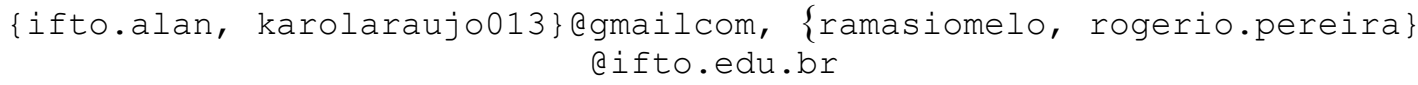

\begin{abstract}
Computational thinking presents itself as a necessary skill for society, thus, this paper addresses an action research in order to analyze the contributions of the S4A course. For the development of computational thinking of students in the 1st grade of a high school in a public school. Seeking to develop cognitive, logical reasoning, individual and collective growth. In class, the contents were works in accordance with the principles of computational thinking, so the results are satisfactory, it was possible to make them more creative, productive, inventive and knowledgeable about how important computing is for men's lives.
\end{abstract}

Resumo. O pensamento computacional apresenta-se como uma habilidade necessária para a sociedade, dessa forma, este trabalho aborda uma pesquisaação com intuito de analisar as contribuições do curso de S4A. Para o desenvolvimento do pensamento computacional dos alunos da $1^{a}$ série do ensino médio de uma escola pública. Buscando desenvolver o cognitivo, raciocínio lógico, crescimento individual e coletivo. Nas aulas, os conteúdos foram trabalhos em conformidade aos princípios do pensamento computacional, assim, os resultados se mostram satisfatórios, foi possível torná-los mais criativos, produtivos, inventivos e conhecedores do quão a computação é importantes para vida dos homens.

\section{Introdução}

A humanidade transformou-se com a chegada dos computadores nos últimos anos. Com o crescimento da indústria tecnológica, a programação de computadores tem-se tornado cada vez mais comum, levando a Ciência da Computação a ser uma das áreas emergentes na atualidade.

Papert Seymour, considerado uma referência no uso de computadores na educação, desenvolveu a Linguagem Logo, que possibilitou aplicação da informática básica na educação, defendendo como uma ideia pode habitar uma mente jovem e as influências do computador nessa perspectiva (PAPERT, 1980). 
VIII Congresso Brasileiro de Informática na Educação (CBIE 2019)

Anais do XXV Workshop de Informática na Escola (WIE 2019)

Wing (2006) conceitua o pensamento computacional (PC) ou computational thinking como sendo uma habilidade essencial que envolve maneiras para resolver problemas, compreender o comportamento humano e projetar sistema embasando-se nos conceitos da Ciência da Computação.

O PC não se restringe apenas às áreas do conhecimento de Ciências Exatas, tendo como princípios norteadores, definidos pelas organizações International Society for Technology in Education (ISTE) e America Computer Science Teachers Association (CSTA): coleta de dados, análise de dados, representação de dados, decomposição de problema, abstração, algoritmos, automação, paralelização e simulação (VALENTE, 2016).

O S4A é uma interface de programação em blocos livre e gratuita, derivada do Scratch, que permite a criação de códigos, para o gerenciamento de sensores e atuadores na plataforma Arduino (S4A, 2015).

Por meio das Tecnologias Digitais de Comunicação e Informação (TDIC) é possível o desenvolvimento de conceitos relacionados ao PC, como exemplo, apresentar problemas e situações, analisando as contribuições e possibilidades que a tecnologia oferece. (VALENTE, 2016).

A utilização do software S4A possui uma interface intuitiva e de fácil manuseio para iniciantes na programação, de maneira a substituir as linhas de código por blocos, organizados por meio de instruções para o Arduino, ao invés de fazer a utilização da sua IDE convencional (KALIL, et al., 2013). Permite ainda, que o aluno possa visualizar o funcionamento de seu código agindo no dispositivo físico, que no caso seria o Arduino.

Ao falar em PC na educação, muitos imaginam logo em computadores nas escolas. No entanto, destaca-se que para desenvolvê-lo, não é necessariamente preciso o uso de computadores, pois existem também as técnicas de computação desplugada.

Santos, et al. (2016) afirma que com essas técnicas é possível estimular o raciocínio e o PC e que além disso, contribui para a criação de ferramentas que possibilitem uma melhor resolução de problemas. Sendo assim, escolas que não possuem este recurso também podem desenvolvê-lo a partir de atividades que irão refletir diretamente na maneira de resolver problemas.

Scolari (2007) coloca que inserir conceitos de Computação pode desenvolver o raciocínio lógico e matemático permitindo tornar os alunos mais críticos nas diferentes disciplinas e, Santos (2016) afirma ainda que os indivíduos tendem a ser mais que apenas meros consumidores de tecnologias, mas sim, produtores.

O PC ainda é pouco explorado na Educação Básica, onde a maioria das aulas ofertadas são de informática básica, com o ensino voltado para utilização dos softwares de escritório, como editores de planilhas e textos. Estes conhecimentos mostram-se defasados para a sociedade contemporânea de tal modo a restringir o crescimento cognitivo do indivíduo com base na teoria do construcionismo (VALENTE, 2016).

Visto que o aperfeiçoamento do PC pode ser mais vantajoso, permitindo que os alunos tenham maior senso crítico e poder para resolução de problemas do cotidiano. Sendo assim, a proposta de estudo e desenvolvimento do curso de S4A apresenta-se relevante fazendo com que os alunos do ensino médio possam estar mais preparados para o futuro. 
VIII Congresso Brasileiro de Informática na Educação (CBIE 2019)

Anais do XXV Workshop de Informática na Escola (WIE 2019)

O PC na educação permite que o indivíduo desenvolva capacidades que contribuem para o melhoramento do raciocínio lógico e habilidades que permitem a solução para resolver problemas (SCAICO, 2013).

Este trabalho apresenta o resultado de um projeto realizado com 12 alunos da $1^{\text {a }}$ série do ensino médio do Colégio da Policia Militar de Araguatins - Unidade VI (CPM VI), onde faz-se a analise da contribuição do curso de S4A para o desenvolvimento do PC destes alunos.

\section{Referencial Teórico}

\subsection{Pensamento computacional}

Para Papert (1980) a programação de computadores além de aperfeiçoar o pensamento e a interação com a realidade possibilita o indivíduo pensar melhor, tornando-se importante para a construção do conhecimento.

No construtivismo de Papert, o computador pode ser apontado como uma ferramenta auxiliadora no desenvolvimento do conhecimento a partir das atividades de controlar e programar (ARANTES; RIBEIRO, 2017).

O PC está ligado diretamente a Ciência da Computação e é considerado uma forma de raciocínio e pensamento sistemático, (SANTOS, et al., 2016; ARANTES; RIBEIRO, 2017) de forma a não se restringir somente a cientistas da computação, mas sim como uma habilidade essencial para todos (WING, 2006).

Cuny, Snyder e Wing (2010, p. 1) definem o PC como "o processo de pensamento envolvido na formulação de problemas e suas soluções, para que suas soluções sejam representadas de forma que possam ser efetivamente realizadas por um agente de processamento de informações".

\subsection{Pensamento computacional na educação básica}

O uso das TDIC na Educação Básica é abordado de diversas maneiras com o intuito de instigar o letramento digital, inclusão da disciplina no currículo e explorar conceitos do PC em jogos e robótica de maneira transversal (VALENTE, 2016).

Autores abordam os aspectos positivos em se trabalhar atividades da Ciência da Computação e ações relacionadas a programação, objetivando o aprimoramento do PC na educação.

Kalil, et al., (2013) relatam uma experiência sobre a robótica educacional no ensino médio analisando quais ferramentas se apresentam mais adequadas para desenvolver a lógica e compreensão do mundo real: Arduino, S4A e Lego. Ressaltam a importância do ensino e aprendizagem de programação, para que a sociedade esteja mais inserida ao universo da computação. Conclusivamente, apresentam o S4A como ferramenta mais adequada, devido sua interface, sintaxe, opção de escolha de idioma e baixo custo.

Zanetti e Oliveira (2015) propõem uma prática pedagógica com a inserção da robótica educacional e do PC para introdução do ensino de programação em um curso técnico de informática integrado ao ensino médio. Trabalharam com três conceitos de programação: entradas e saídas, estrutura condicional e de repetição para desviar o robô de um obstáculo utilizando o S4A e componentes do Arduino. As resultantes do trabalho mostram que a prática abordada torna um ambiente de aprendizagem menos abstrato para 
VIII Congresso Brasileiro de Informática na Educação (CBIE 2019)

Anais do XXV Workshop de Informática na Escola (WIE 2019)

o aluno, a fim de que, o aluno venha a ser mais ativo na construção do conhecimento e do raciocínio lógico.

Albuquerque et al. (2016), relatam um experimento com alunos do curso técnico em mecatrônica integrado ao ensino médio, onde utilizou para o ensino de programação, a plataforma Arduino e o PC, de modo que eles construíssem em duplas um projeto de eletrônica do seu cotidiano. Os autores constataram que os alunos não tiveram grandes dificuldades em trabalhar com o Arduino, a não ser, devido ao idioma Inglês. Destacaram ainda que a metodologia do experimento é mais apropriada do que a metodologia tradicional por apresentar uma aprendizagem menos abstrata, e foi possível desenvolver o PC por relacionar um problema da vida real sendo resolvido pelos alunos por intermédio dos conceitos de programação e do Arduino.

Pereira e Franco (2018) utilizaram o S4A para desenvolver o PC com alunos do $7^{\circ}, 8^{\circ}$ e $9^{\circ}$ ano do ensino fundamental. Abordaram conteúdos de introdução a programação como: hardware e software, lógica e algoritmo. Utilizaram o S4A para desenvolver projetos que foram apresentados em uma feira de ciências pelos alunos. Os resultados elencados demonstram que foi possível relacionar os conhecimentos abstratos aos concretos, com a verificação dos códigos agindo sobre o hardware e os desafios impostos aos alunos induzindo-os a realizarem buscas de soluções, assim, influenciando no modo de aprendizagem e desenvolvimento do PC dos alunos.

A necessidade de uma educação que oportunize o desenvolvimento de jovens em adultos mais competentes, também se atrela a utilização dos diferentes instrumentos que o ensino da Computação e o uso das tecnologias podem ser usados a favor não apenas do entretenimento ou diversão, mas, para torná-los capazes de aprender e ter autonomia em resolver problemas exercitando assim o PC (SCAICO, 2013).

\subsection{S4A e robótica educacional}

Oriundo do Scratch, o S4A é uma adaptação para se programar de forma simplificada a plataforma Arduino, o que consequentemente pode atrair maior público para o mundo da programação. Sua interface continuou similar à do Scratch, exceto por adicionar blocos de ações para gerenciamento de sensores e atuadores. O S4A foi desenvolvido no Citilab pelo Edutec Research Group, com a ajuda do grupo de programação Smalltalk.cat e de colaboradores como Jorge Gómez e atualmente está na versão 1.3 (S4A, 2015).

Hernandez (2013) cita que o S4A pode viabilizar a interação com a plataforma Arduino e permitir maior usabilidade, substituindo sua IDE padrão baseada em Processing.

Arduino é uma plataforma de prototipação, sendo uma placa física de distribuição livre. Nos permite conectar diversos sensores e atuadores como por exemplo, sensor de temperatura, luminosidade, umidade, distância e outros como LED, motores, Bluetooth, etc (ARDUINO, 2015).

\section{Procedimentos Metodológicos}

A presente pesquisa possui caráter exploratório, tal como GIL (2008) relata que esta categoria tem como finalidade proporcionar ao pesquisador maior intimidade com o problema, tornando-o mais aparente, possibilitando o melhoramento de ideias. Como também está sendo caracterizada de pesquisa-ação, onde o pesquisador está inserido no contexto da pesquisa, participando e interagindo com a situação ou problema que pretende 
VIII Congresso Brasileiro de Informática na Educação (CBIE 2019)

Anais do XXV Workshop de Informática na Escola (WIE 2019)

intervir (GIL, 2008).

Nessa perspectiva, o trabalho se dará em quatro passos: planejamento das atividades, intervenção com o curso proposto, observação junto a um questionário como meio para avaliação, e a reflexão analisando os dados, as habilidades, as vantagens e as melhorias para o curso.

A pesquisa possui abordagem quali-quantitativa, onde os instrumentos para coleta de dados serão o de observação e questionário fechado. O público alvo são alunos da $1^{\mathrm{a}}$ série do ensino médio do CPM VI, correspondentes a uma faixa etária entre 14 a 16 anos de idade. O curso foi ofertado aos alunos que demonstraram interesse na temática compondo uma turma de 12 alunos de acordo com capacidade do Laboratório Móvel da Rede e-Tec Brasil.

As aulas aconteceram em encontros semanais com duração de $4 \mathrm{~h} /$ aulas. $\mathrm{O}$ curso de S4A foi aplicado e distribuído nas seguintes etapas: PC.

$1^{\text {o }}$ etapa: apresentação da plataforma S4A, do Arduino e das estruturas básicas do

$2^{\circ}$ etapa: manuseio da plataforma S4A com o Arduino de forma prática, desenvolvendo a lógica de programação e animação, explorando o funcionamento da plataforma de prototipagem e verificando o gerenciamento dos sensores/atuadores a partir da criação de mini projetos assistidos para facilitar o entendimento dos alunos sobre o funcionamento básico das ferramentas utilizadas.

$3^{0}$ etapa: composta principalmente pelas propostas dos alunos para a construção do protótipo, onde foram divididos em 4 grupos de 3 alunos e cada grupo trabalhou de maneira colaborativa na construção, organização e abstração do problema, para que chegassem as possíveis soluções.

$4^{\circ}$ etapa: composta essencialmente do desenvolvimento dos protótipos, onde os grupos definidos construíram, com base na $3^{\text {a }}$ etapa, objetivando a solução de algum problema da sociedade.

Após a $4^{\circ}$ etapa, houve coleta de dados que dividiu-se em duas partes: análise dos projetos desenvolvidos e o questionário Survey usando a escala Likert aplicado aos alunos do curso.

\section{Resultados e Discussões}

Foi possível constatar na ambientação que os alunos se mostraram bastante entusiasmados a contribuir para o desenvolvimento dos projetos assistidos (figura 1). 
VIII Congresso Brasileiro de Informática na Educação (CBIE 2019)

Anais do XXV Workshop de Informática na Escola (WIE 2019)

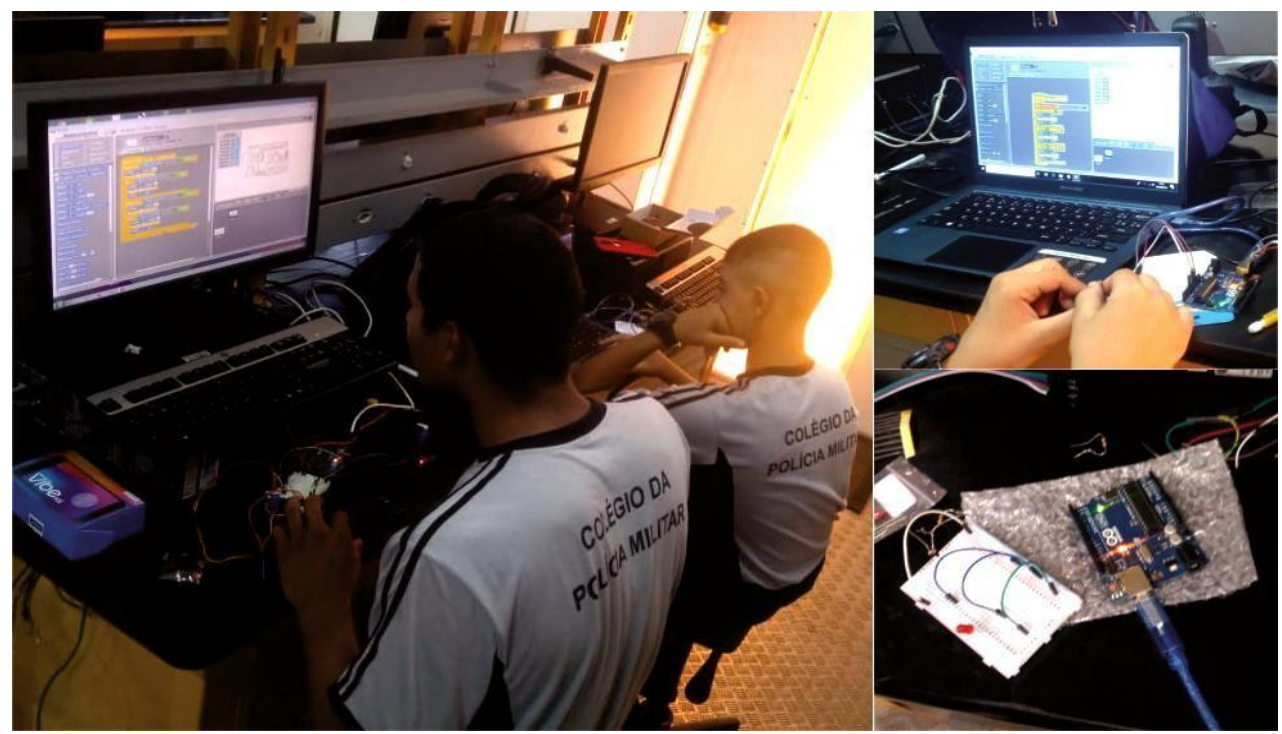

Figura 1. Desenvolvimento dos protótipos

As ferramentas S4A e o Arduino rapidamente possibilitaram o desenvolvimento das habilidades como: controlar sensores e atuadores, entender razoavelmente a lógica de programação e funcionamento do Arduino, constatando ser uma ferramenta muito útil para projetos simples.

Os alunos analisaram vários problemas do seu cotidiano e apresentaram as propostas de projetos para a turma, visando a solução para alguma necessidade da escola. O projeto selecionado consistiu-se em um protótipo para gerenciar um estacionamento, fragmentado em 4 partes e distribuídas em: (a) carro, (b) semáforo (c) controle de acesso, (d) controle de vagas.

$\mathrm{Na}$ etapa do desenvolvimento os alunos partiram para a programação no S4A e para a construção de uma maquete como proposta de solução, logo após integraram as partes do projeto e apresentaram na escola concedente, na II Semana de Popularização da Ciência do IFTO - Campus Araguatins e em praça pública para a comunidade (figura 2).

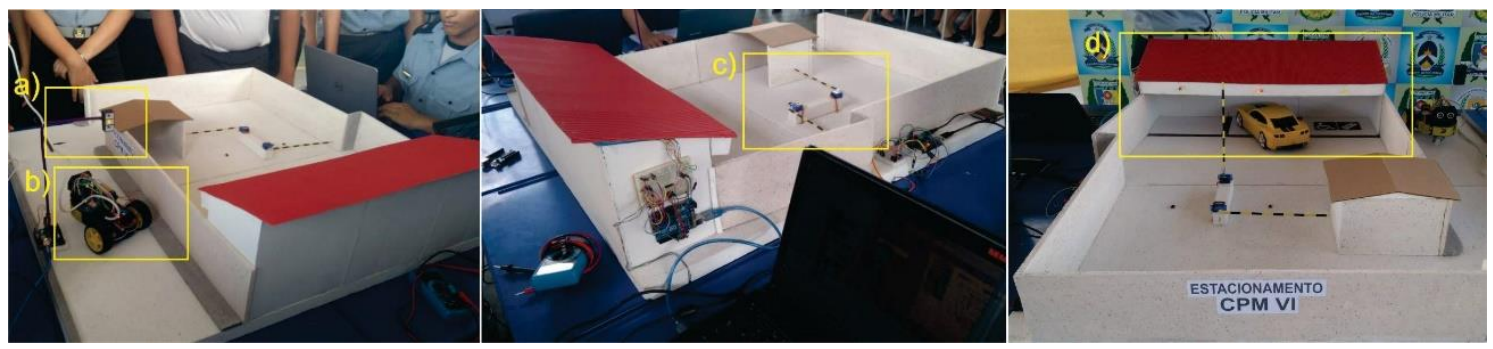

Figura 2: Maquete do estacionamento.

O curso finalizou com total de 4 alunos devido a escola possuir um sistema rigoroso de dedicação e comprometimento, levando alguns alunos a desistiram por não conseguirem conciliar as atividades escolares com as atividades do curso, mostrando-se desfavoráveis para a aplicação do mesmo.

Foi proposto um questionário aos quatro (4) alunos participantes, em que os quatro (4) se disponibilizaram a responder. O questionário era composto de oito (8) questões, 
VIII Congresso Brasileiro de Informática na Educação (CBIE 2019)

Anais do XXV Workshop de Informática na Escola (WIE 2019)

mas somente quatro (4) questões foram selecionadas por serem consideradas de maior relevância para o trabalho.

Inicialmente questionou-se sobre o desenvolvimento dos conceitos e habilidades de programação por meio dos projetos desenvolvidos durante o curso. Pôde-se constatar que $(80 \%)$ dos alunos concordam totalmente e $(20 \%)$ parcialmente. Dessa forma, o curso demonstra ter contribuído para o aprimoramento da aprendizagem sobre lógica de programação.

Os alunos desenvolveram inicialmente projetos assistidos fazendo com que entendessem a estruturação e o funcionamento da programação em blocos, habituandose a interface do S4A. Conceitos e habilidades para estabelecer variáveis, aplicar estrutura condicional de repetição e lógica de programação representam uma forma introdutória fundamental na estruturação de instruções para resolução de problemas, sendo estes conhecimentos essenciais para o desenvolvimento do PC (ZANETTI; OLIVEIRA, 2015).

Quando questionados a respeito da definição das etapas para se chegar nas soluções dos problemas propostos, (80\%) concordaram totalmente e (20\%) parcialmente.

A decomposição de problemas faz parte dos princípios norteadores do PC, esta habilidade de fragmentar o problema é importante por torná-lo mais simples e de fácil percepção. Após definição projeto, os alunos foram organizados em grupos e a eles foi destinado algumas tarefas. Cada grupo apresentou o planejamento de seu protótipo, para isso tiveram que realizar pesquisas na internet a fim de responder algumas questões como: Quais recursos seriam necessários para a construção do projeto? Quais ações seu projeto deveria executar? Quais instruções seriam necessárias para o projeto?

A lógica de programação possibilita ao aluno aprimorar suas habilidades, ao estruturar um problema o indivíduo terá segmentando-o para resolvê-los de forma simplória (BARBOSA, 2017).

Questionou-se aos alunos se as partes do projeto desenvolvidos estão relacionados a problemas do cotidiano, $(100 \%)$ concordaram totalmente, o que revela ser algo positivo, pois permitiu que eles trabalhassem problemas reais, a fim de uma melhor contextualização e aplicação da computação no dia-a-dia.

É importante integrar os princípios do PC na educação básica, pois fornece estruturas para o desenvolvimento cognitivo do aluno objetivando a resolução de problemas em diferentes áreas do conhecimento (Brasil, 2012). Desse modo, pôde-se formar alunos mais ativos e críticos no meio social, dando a oportunidade de resolver os problemas de forma sistemática e contextualizada.

Uma questão subjetiva foi disponibilizada aos alunos em relação às dificuldades que surgiram durante o curso. A maioria dos alunos relatou dificuldades no início do curso por não terem contato ou conhecimento prévio sobre lógica de programação e Arduino. Se deu ao fato que seria a primeira experiência com essas tecnologias, mas no decorrer do curso foi possível saná-las. Outra dificuldade apontada pelos participantes está relacionada à decomposição de problemas (divisão dos problemas em partes menores).

\section{Considerações Finais}

O presente trabalho abordou uma proposta metodológica para o desenvolvimento do PC 
VIII Congresso Brasileiro de Informática na Educação (CBIE 2019)

Anais do XXV Workshop de Informática na Escola (WIE 2019)

através do curso de introdução ao S4A, desenvolvendo o ensino de lógica de programação e protótipos com o intuito de analisar as contribuições das ferramentas utilizadas para o aperfeiçoamento do PC.

Constatou-se que o curso de introdução ao Scratch for Arduino foi uma boa iniciativa para o desenvolvimento do PC na educação por permitir maior concretude e autonomia no aprendizado dos alunos. Os projetos foram trabalhados em conformidade aos princípios do PC, possibilitando o desenvolvimento dos conceitos e habilidades de programação e Arduino, na decomposição de problemas, facilitando suas soluções, além de contextualizá-los. Algumas dificuldades surgiram devido à inexperiência no manuseio das ferramentas, porém foram sanadas ao decorrer do curso.

A pesquisa apresentou importantes contribuições a respeito da inserção do PC na educação, visto que a sociedade tende a estar cada vez mais ligada às tecnologias e que desenvolver o PC é de extrema relevância para a participação de maneira efetiva nesta sociedade.

Para trabalhos futuros, pretende-se ofertar cursos baseados nos princípios de PC para turmas maiores e diversificar as atividades e agregar outras ferramentas ao curso para fins de comparação e contribuição para integração do PC na educação.

\section{Referências}

ALBUQUERQUE, David et al. Uma Experiência do Uso Do Hardware Livre Arduino no Ensino De Programação De Computadores. In: Anais do Workshop de Informática na

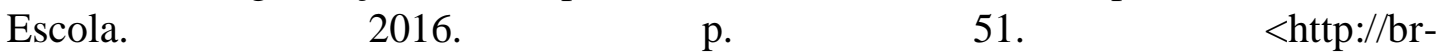
ie.org/pub/index.php/wie/article/download/6602/4513>. Acesso em: 29 maio 2018.

ARANTES, Flávia Linhalis; RIBEIRO, Paula Eduarda Justino. Desenvolvimento do Pensamento Computacional com Valores da Ética Hacker. Informática na educação: teoria \& prática, v. 20, n. 2 mai/ago. Disponível em:

$<$ https://scholar.google.com.br/scholar?hl=ptBR\&as_sdt=0\%2C5\&q=desenvolvimen to+do+pensamento+computacional+com+val ores+da+\%C3\%A9dica+hacker\&btnG=>. Acesso em: 29 maio 2018.

ARDUINO. Disponível em: <www.arduino.cc/>. Acesso em: 27 maio 2018.

BARBOSA, Débora Nice Ferrari et al. ENSINANDO LÓGICA COM AS TECNOLOGIAS DA INFORMAÇÃO: DESENVOLVENDO O RACIOCÍNIO LÓGICO E O PENSAMENTO COMPUTACIONAL. CATAVENTOS-Revista de Extensão da Universidade de Cruz Alta, v. 9, n. 1, p. 54-72, 2017.

CUNY, J.; SNYDER, L.; WING, Jeannette M. Demystifying computational thinking for non-computer scientists. Unpublished manuscript in progress, 2010. Disponível em: http://www.cs.cmu.edu/ CompThink/resources/TheLinkWing.pdf. Acesso em: 28 maio 2018.

GIL, Antonio Carlos. Métodos e técnicas de pesquisa social. 6. ed. - São Paulo: Atlas, 2008. Disponível em: <https://ayanrafael.files.wordpress.com/2011/08/gil-acmc3a9todos-e-tc3a9cnicas-de-pesquisa-social.pdf>. Acesso em: 28 maio 2018.

KALIL, Fahad. et al. Promovendo a robótica educacional para estudantes do ensino médio público do Brasil. Nuevas Ideas en Informática Educativa, TISE, p. 739-742, 2013. 
VIII Congresso Brasileiro de Informática na Educação (CBIE 2019)

Anais do XXV Workshop de Informática na Escola (WIE 2019)

Disponível em: <http://www.tise.cl/volumen9/TISE2013/739-742.pdf>. Acesso em: 28 maio 2018.

PAPERT, S. Mindstorms: Children, computers, and powerful ideas. New York, NY: Basic Books,1980. Disponível em: $<$ http://worrydream.com/refs/Papert\%20\%20Mindstorms\%201st\%20ed.pdf $>$. Acesso em: 28 maio. 2018.

PEREIRA, Amanda C.; FRANCO, Matheus E.. Desenvolvendo o pensamento computacional no ensino fundamental com Arduino e Scratch. In: ENCONTRO NACIONAL DE COMPUTAÇÃO DOS INSTITUTOS FEDERAIS (ENCOMPIF/CSBC), 5. , 2018, 1/2018. $5^{\circ}$ Encontro Nacional de Computação dos Institutos Federais (ENCompIF 2018). Porto Alegre: Sociedade Brasileira de Computação, july 2018. Disponível em: <http://portaldeconteudo.sbc.org.br/index.php/encompif/article/view/3561>. Acesso em: 22 dez. 2018.

S4A. Disponível em: <http://s4a.cat/>. Acesso em: 21 maio. 2018.

SANTOS, Elisângela Ribas dos et al. Estímulo ao Pensamento Computacional a partir da Computação Desplugada: uma proposta para Educação Infantil. 2016. Disponível em: <http://dehesa.unex.es/handle/10662/5939>. Acesso em: 28 maio. 2018.

SCAICO, Pasqueline Dantas et al. Ensino de programação no ensino médio: Uma abordagem orientada ao design com a linguagem scratch. Revista Brasileira de Informática na Educação, v. 21, n. 02, p. 92, 2013. Disponível em: <http://www.academia.edu/download/43035180/2364-4111-1-PB.pdf >. Acesso em: 28 maio. 2018.

SCOLARI, T. Angélica; BERNARDI, Giliane; CORDENONSI, Z. Andre. O Desenvolvimento do Raciocínio Lógico através de Objetos de Aprendizagem. Santa Maria - $\quad$ RS, $2007 . \quad$ Disponível em: <http://www.cinted.ufrgs.br/ciclo10/artigos/4eGiliane.pdf $>$. Acesso em: 22 de maio de 2016.

VALENTE, José Armando. Integração do pensamento computacional no currículo a Educação Básica: diferentes estratégias usadas e questões de formação de professores e avaliação do aluno. Revista e-Curriculum, v. 14, n. 3, p. 864-897, 2016. Disponível em: <http://www.redalyc.org/pdf/766/76647706006.pdf>. Acesso em: 23 maio. 2018.

WING, Jeannette M. Computational thinking. Communications of the ACM, v. 49, n. 3, p. 33-35, 2006. Disponível em: <https://www.cs.cmu.edu/ 15110s13/Wing06ct.pdf>. Acesso em: 28 maio. 2018.

ZANETTI, Humberto; OLIVEIRA, Claudio. Práticas de ensino de Programação de Computadores com Robótica Pedagógica e aplicação de Pensamento Computacional. In: Anais dos Workshops do Congresso Brasileiro de Informática na Educação. 2015. p. $1236 . \quad$ Disponível em: <https://brie.org/pub/index.php/wcbie/article/view/6268/4389>. Acesso em: 19 jul. 2019 